\title{
Medial Prefrontal Cortex Activity during the Extinction of Conditioned Fear: An Investigation Using Functional Near-Infrared Spectroscopy
}

\author{
Anne Guhn $^{a}$ Thomas Dresler ${ }^{a, b}$ Tim Hahn ${ }^{a, c}$ Andreas Mühlberger ${ }^{d}$ \\ Andreas Ströhle $^{\mathrm{e}}$ Jürgen Deckert ${ }^{\mathrm{a}}$ Martin J. Herrmann ${ }^{\mathrm{a}}$ \\ aDepartment of Psychiatry, Psychosomatics and Psychotherapy, University of Würzburg, Würzburg, \\ ${ }^{b}$ Department of Psychiatry and Psychotherapy, University of Tübingen, Tübingen, ' Department of Cognitive \\ Psychology II, University of Frankfurt/Main, Frankfurt, dDepartment of Psychology, University of Würzburg, \\ Würzburg, e'Department of Psychiatry and Psychotherapy, Campus Charité Mitte, Charité - Universitätsmedizin \\ Berlin, Berlin, Germany
}

\section{Key Words}

Extinction learning $\cdot$ Fear conditioning $\cdot$ Medial prefrontal cortex $\cdot$ NIRS

\begin{abstract}
The majority of fear conditioning studies in humans have focused on fear acquisition rather than fear extinction. For this reason only a few functional imaging studies on fear extinction are available. A large number of animal studies indicate the medial prefrontal cortex (mPFC) as neuronal substrate of extinction. We therefore determined mPFC contribution during extinction learning after a discriminative fear conditioning in 34 healthy human subjects by using functional near-infrared spectroscopy. During the extinction training, a previously conditioned neutral face (conditioned stimulus, CS+) no longer predicted an aversive scream (unconditioned stimulus, UCS). Considering differential valence and arousal ratings as well as skin conductance responses during the acquisition phase, we found a CS+ related increase in oxygenated haemoglobin concentration changes within the MPFC over the time course of extinction. Late CS+ trials further revealed higher activation than CS- trials in a cluster of probe set channels covering the MPFC. These results are in line with
\end{abstract}

previous findings on extinction and further emphasize the mPFC as significant for associative learning processes. During extinction, the diminished fear association between a former CS+ and a UCS is inversely correlated with mPFC activity - a process presumably dysfunctional in anxiety disorders.

Copyright $\odot 2012$ S. Karger AG, Basel

\section{Introduction}

Fear is an aversive emotional state which at moderate levels proves biologically useful by enabling effective detection of threat and automatic activation of defensive behaviour [1]. Anxiety disorders such as post-traumatic stress disorder, panic disorder and phobias are characterized by increased fear levels that might contribute to a generally impaired ability of fear extinction [2-4]. In order to model the development and maintenance of anxiety disorders, learning theories - notably conditioned fear reactions and their extinction - have been widely applied and particularly validated with regard to the effectiveness of exposure-based treatment in psychotherapy [2].

\section{KARGER \\ Fax +4161306 1234 \\ E-Mail karger@karger.ch}

www.karger.com
(C) 2012 S. Karger AG, Basel

0302-282X/12/0654-0173\$38.00/0

Accessible online at:

www.karger.com/nps
Anne Guhn

Department of Psychiatry, Psychosomatics and Psychotherapy

University of Würzburg, Füchsleinstrasse 15

DE-97080 Würzburg (Germany)

Tel. +49 931201 77440, E-Mail Guhn_A@klinik.uni-wuerzburg.de 
In a classical pavlovian fear conditioning paradigm, an initially neutral stimulus, such as a tone, is paired with an aversive event (unconditioned stimulus, UCS), e.g. an electric shock, and comes to elicit the so-called conditioned response (CR, e.g. freezing) itself after several pairings. In the absence of the UCS, the CR gradually disappears in response to the conditioned stimulus (CS, tone), i.e. its amplitude and frequency decrease [2]. This decrease of the CR is referred to as extinction and is thought to be the experimental foundation of exposure therapy applied to anxiety patients. During extinction learning, the acquisition of inhibitory memories is assumed to compete with excitatory memories formed during fear conditioning and thereby suppress the CR $[3,4]$. These interactions are neuroanatomically mirrored in subcortical as well as cortical structures which have been closely investigated in a variety of lesion studies [e.g. 5, 6] as well as single-cell recording [e.g. 7, 8] and stimulation studies [e.g. 9, 10] in animals. Above all, the amygdala has been shown to be notably involved in the expression and acquisition of conditioned fear [11], also in humans [12]. In intracellular in vivo recordings in rats, Rosenkranz et al. [7] demonstrated enhanced activity of the lateral amygdala while presenting conditioned affective stimuli whereas bilateral amygdala lesions prevented the acquisition of CR $[13,14]$. Lesions to the medial prefrontal cortex (mPFC) on the other hand have been shown to generate an increased resistance to extinction as well as a high rate of spontaneous recovery while the acquisition of fear CR remained unaffected $[6,15,16]$. During extinction learning a rapid switch in the activity of two distinct populations of basal amygdala neurons seems to be essential for activating behavioural alterations [8]. According to Herry et al. [8], these 'extinction neurons' are bi-directionally connected with $\mathrm{mPFC}$ neurons that might mediate the consolidation of extinction memory. The 'fear neurons' in turn seem to be depressed if the predictive ability of the CS for danger is weakened through successful extinction training [5]. Paralleling these findings, mice that underwent extinction training in an investigation using fluorodeoxyglucose displayed elevated prefrontal cortex activity. Moreover, mice with higher prefrontal activity showed less $\mathrm{CR}$ and in turn mice not receiving extinction training demonstrated significantly more stable amygdala activity [17].

Although the neural mechanisms of extinction learning in humans are less well characterized than for animals [12], the general pattern of brain activation during fear and extinction learning seems to be essentially the same [18]. A variety of pavlovian conditioning studies in humans actually found amygdala involvement during fear conditioning [19-27]. Accordingly, mPFC activity could have been associated with extinction learning [20, $23,24,26,28]$ and further linked with decreasing amygdala activity [23, 24, 29-31]. However, although extinction is thought to be crucial for understanding and improving psychotherapy, in 2009, Sehlmeyer et al. [12] merely found seven studies directly focussing on neurobiological correlates of extinction learning. Frequently but even inconsistently found was prefrontal engagement. More recently these findings were enriched by studies concentrating on trait anxiety in fear extinction. These studies revealed comparable results to the aforementioned animal studies $[7,10]$ relating to $\mathrm{mPFC}$-amygdala coupling. Anxious participants displayed enhanced amygdala activity during extinction learning that correlated negatively with mPFC involvement, indicating delayed inhibitory learning or rather generally reduced extinction $[26,28]$. Irrespective of extinction learning, in anxious subjects, Indovina et al. [32] demonstrated an insufficient recruitment of the prefrontal cortex to downregulate fear in a safety context, and Bishop et al. [33] showed reduced $\mathrm{mPFC}$ activity when anticipating threat. Taken together, these findings clarify extinction as a form of new learning which is hence prone to behavioural instability and emphasise the importance of a better understanding of extinction mechanisms. To date, the limited number of available studies on mPFC activity and extinction learning in humans impedes gathering insides into the mode of action in psychotherapy.

The present investigation therefore focused on contribution of the mPFC during extinction learning in a classical discriminative fear conditioning paradigm by using functional near-infrared spectroscopy (fNIRS). NIRS is an optical imaging method to non-invasively and in vivo investigate tissue such as the brain, muscle and others. It enables measuring concentration changes in oxygenated $\left(\mathrm{O}_{2} \mathrm{Hb}\right)$ and deoxygenated $(\mathrm{HHb})$ haemoglobin which are accompanied by increases in cerebral blood volume [34]. To our knowledge, fNIRS has never been used to investigate contribution of the mPFC to extinction learning. However, fNIRS has been successfully applied to measure changes in $\mathrm{O}_{2} \mathrm{Hb}$ concentration within the $\mathrm{mPFC}$ during emotional tasks [e.g. 35]. The frontal positioning of the NIRS probe set enables the investigation of medial Brodmann areas 9 and 10 bilaterally extending to the dorsolateral prefrontal cortices and is hence covering the mPFC. Participants in the present study performed a fear conditioning paradigm in which one of two neutral faces (CS) was paired with an aversive scream (UCS). Immedi- 
ately after establishing the fear conditioning they underwent an extinction training in which the originally neutral stimulus was repeatedly presented without the UCS. By dividing the so-called within-session extinction phase into an early and late component, we hypothesised increasing $\mathrm{mPFC}$ activity to the CS+ as described by others $[25,26,36]$.

\section{Methods}

\section{Participants}

Thirty-five healthy volunteers (17 females, 18 males; mean age 24.7 years, standard deviation (SD) 3.32, range 20-32 years) participated in the study. All subjects were screened for current mental health using the German version of the Mini International Neuropsychiatric Interview (MINI [37]) and for right-handedness according to the Edinburgh Handedness Inventory [38] before the experiment. We further assured that all females used oral monophasic contraceptives and that they were not in their pill-off phase when participating in the experiment in order to exclude changes in hormonal levels which have been demonstrated to influence conditioned fear acquisition as well as extinction recall [39]. Psychology students were excluded to exclusively investigate paradigm-naive volunteers. For this reason, 1 male participant (age 31 years) was not considered for further data analyses because of familiarity with the procedure. Subjects were reimbursed with $7 \mathrm{Eu}$ ros for participation in an experimental setting lasting $60 \mathrm{~min}$.

They were recruited through online advertisement and gave written informed consent in accordance with the Declaration of Helsinki in their most recent version from 2008. All procedures were approved by the ethical review board of the medical faculty of the University of Würzburg (Protocol ID 151/10) and were performed in the facilities of the department of Psychiatry, Psychosomatics and Psychotherapy of the University of Würzburg.

\section{Experimental Paradigm}

The differential fear conditioning paradigm investigated in the present study consisted of three experimental phases (habituation, acquisition and extinction). Two colour photographs of neutral male faces selected from the NimStim set of facial expressions [40] served as conditioned stimuli (CS) and a scream of 95 $\mathrm{dB}$ adapted from the International Affective Digital Sounds [41] was used as UCS. During the habituation, each stimulus was presented 8 times without the UCS. The following acquisition phase comprised 30 trials in total, i.e. $15 \mathrm{CS}-$ and $15 \mathrm{CS}+$ trials in which $12 \mathrm{CS}+$ were paired with the UCS and the remaining $3 \mathrm{CS}+$ trials rested unpaired (reinforcement rate $80 \%$ ) in order to decelerate the acquisition of conditioned fear and to extend its extinction. The CS- was never paired with the UCS. The extinction phase consisted of $18 \mathrm{CS}-$ and $18 \mathrm{CS}+$ presentations without the UCS. Faces were presented for 4,000 ms and counterbalanced as CS+ and CS- to each subject, so that both faces were equally often selected as CS+ and CS-. The scream lasted 1,380 ms and appeared in a jittered time interval of $0-1,000 \mathrm{~ms}$ after the CS+ offset (fig. 1). Inter-trial intervals ranged from 10 to $16 \mathrm{~s}$ and consisted of a white fixation cross on a black screen. CS presentations were randomized within all three experimental phases.

Medial Prefrontal Cortex Activity during the Extinction of Conditioned Fear

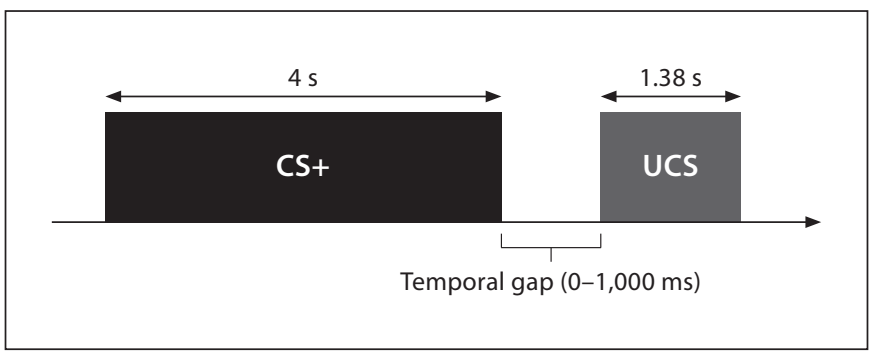

Fig. 1. Temporal arrangement of CS+ and UCS.

In order to adjust the NIRS probe set to cover concentration changes in cerebral haemoglobin while passing through the extinction, we implemented a short break after the acquisition phase lasting 2-4 min. We did not change any contextual parameter such as the lighting conditions or the subjects' sitting position to ensure within-session extinction. All subjects were informed that during the experiment one of two male faces is paired with an aversive loud scream, whereas the second face is never presented together with a scream. Apart from their knowledge of the break to adjust the NIRS probe set, they were not informed about the experimental phases and in particular the UCS absence during the extinction phase. In order to eliminate intense novelty responses to the UCS and thereby risking dropouts during the paradigm, subjects were presented once with the scream before the experiment and were asked to determine the level of aversiveness on a scale ranging from 0 for 'not unpleasant' to 10 for 'extremely unpleasant'. While passively regarding the faces during the examination, subjects had to field a total of seven valence and arousal ratings of the CS to maintain attention and ensure successful conditioning over the whole experiment, i.e. approximately 30 min: after the habituation phase and 3 times during acquisition and extinction, respectively. Therefore, we used the Self-Assessment Manikin [42] and a 9-point Likert Scale ranging from 1 for 'unpleasant' to 9 for 'pleasant' and 1 for 'not arousing' to 9 for 'highly arousing'. We additionally assessed contingency awareness on CS+ and UCS and implemented three expectancy ratings during the extinction phase. Expectancy ratings were formulated as 'How likely is a reappearance of the scream in your opinion?' and were assessed by a 9-point Likert Scale ranging from 0 to $100 \%$ conviction of UCS recurrence.

These expectancy ratings are expected to represent a kind of prediction error, i.e. the higher the prediction error was, the higher the expectation of recurring UCS towards the following items is. The paradigm was presented using Presentation ${ }^{\circledR}$ Version 12.2 software (Neurobehavioral Systems, Inc., Albany, Calif., USA). All responses were given by keyboard presses with the right hand.

\section{SCR and NIRS Measurements}

We assessed skin conductance responses (SCR) to CS+ and CS-during the whole experiment in order to ensure effective fear conditioning as well as extinction. SCR is regarded as an index for emotional responses associated with automatic arousal [43] and therefore an indicator of successful conditioning. It was assessed with two $\mathrm{Ag} / \mathrm{AgCl}$ electrodes attached to the thenar eminence of the subjects' left palm. SCR were recorded using a GSR sensor 
(Brain Products $\mathrm{GmbH}$, Munich, Germany) constantly delivering $0.5 \mathrm{~V}$ across both electrodes and a 72-channel QuickAmp amplifier (Brain Products $\mathrm{GmbH}$ ) with a sampling rate of $1,000 \mathrm{~Hz}$ and a notch filter of $50 \mathrm{~Hz}$. Data were acquired and saved via Vision Recorder Version 2.0 software (Brain Products $\mathrm{GmbH}$ ) and towards data collection processed by the appropriate Vision Analyzer software (Brain Products $\mathrm{GmbH}$ ). Herewith, time series were filtered at $1 \mathrm{~Hz}$ and segmented into experimental phases as well as single CS+ and CS - trials. Each segment was further baseline corrected 1,000 ms prior to the onset of the stimuli and characterised by the peak response of the SCR signal between 1 and $4 \mathrm{~s}$ after stimulus onset. Artefact rejection was conducted manually for all 82 trials per subject.

In order to investigate regional cerebral blood flow in the mPFC during extinction learning, we examined changes in $\mathrm{O}_{2} \mathrm{Hb}$ by using fNIRS. fNIRS measurements are based on differential absorption of near-infrared light due to $\mathrm{O}_{2} \mathrm{Hb}$ and $\mathrm{HHb}$ concentration changes that arise through neurovascular coupling mirroring the metabolic demands of the nervous system. Illuminating the brain surface through the intact scalp and skull, near-infrared light reflected from deep tissue layers is received by a photodetector that is fixed some centimetres apart from the light emitter. fNIRS measurements are comfortable for the subjects because of fewer motion restrictions and no noise disturbance; it has a high temporal resolution $(<1 \mathrm{~s})$ and can be easily combined with other neuroimaging techniques or physiological measurements [44]. Further, more detailed information about the fundamentals of fNIRS is provided elsewhere [e.g. 45, 46]. We opted to restrict the fNIRS measurement to the extinction phase for two reasons: first, our experiment focused particularly on extinction learning and not fear acquisition, and second, we know from experience that subjects in fNIRS settings exceeding a time period of 20 min without a break might feel more and more uncomfortable. fNIRS signals were measured with the continuouswave system ETG-4000 (Hitachi Medical Co., Tokyo, Japan) using a $3 \times 11$ channel array of optodes consisting of 16 photodetectors and 17 light emitters resulting in 52 channels in total. The ETG-4000 operates with two different wavelengths $(695 \pm 20$ and $830 \pm 20 \mathrm{~nm}$ ) and its frequency is modulated for wavelengths and channels to prevent crosstalk. In order to reliably position the probe set, the lowest-row centre optode is typically placed on the Fpz position at the frontal region of the head extending symmetrically towards positions T3 and T4 according to the International 10-20 system for EEG electrode placement [47]. The interoptode distance of $30 \mathrm{~mm}$ enables measurements approximately $15-25 \mathrm{~mm}$ beneath the scalp [48]. Signals were acquired with a sampling rate of $10 \mathrm{~Hz}$ and transformed into values for changes in the concentration of $\mathrm{O}_{2} \mathrm{Hb}$.

\section{Statistical Methods}

All statistical analyses were performed using PASW Statistics 18 (SPSS Inc., Chicago, Ill., USA) and Matlab software (Version 7; MathWorks Inc., Natick, Mass., USA). Whenever we had directed hypotheses, one-tailed tests at a significance level of $\mathrm{p}<0.05$ were performed (otherwise two-tailed). Valence and arousal ratings were analysed separately using repeated-measures analyses of variance (ANOVA) with two within-subject factors: stimulus (CS+, CS-) and phase (habituation, acquisition, extinction). For the repeated ratings derived from the acquisition and extinction phases, values were averaged. In case of significant stimulus $\times$ phase interactions post hoc $\mathrm{t}$ tests were performed. Non-sphericity was considered applying Greenhouse-Geisser correction.

Before performing statistical analyses for the SCR data, we logtransformed all peak amplitudes $(\mathrm{SCR}+1)$ to normalize the distribution and further scored responses $<0 \mu \mathrm{S}$ as zero in order to adequately characterize non-responses to the CS. Afterwards we separated the existing $18 \mathrm{CS}+$ and $18 \mathrm{CS}$ - extinction trials into early $(n=9)$ and late $(n=9)$ responses to compare SCR and fNIRS parameters. SCR data were analysed with repeated-measures ANOVA with stimulus (CS+, CS-) and phase (habituation, acquisition, early extinction, late extinction) as within-subject factors. A significant interaction of both factors was further assessed with one-tailed post hoc t tests at a significance level of $\mathrm{p}<0.05$ due to our directed hypotheses in fear and extinction learning. In relation to our assumptions concerning the fNIRS signal changes, we expected that successful extinction will be indicated by a decrease in SCR to the CS+ during the time course of extinction training.

Analogous to the procedure for SCR analyses, fNIRS signals were divided into an early and late phase, each consisting of nine trials. Because of our interest in signal changes occurring in response to the CS+ offset and accordingly the anticipated UCS onset, all trials were time-locked to the jitter mean, i.e. 4,500 ms after $\mathrm{CS}+$ onset, and screened for artefacts. $\mathrm{O}_{2} \mathrm{Hb}$ changes were preprocessed by applying a low-pass filter of $0.5 \mathrm{~Hz}$ and a cosine filter correcting for low-frequency signal drifts. In a next step, functional data were modelled by four regressors (CS+ early, CSearly, CS+ late, CS- late) (online supplementary fig. 1; see www. karger.com/doi/10.1159/000337002). Events per condition were further modelled as $\delta$ functions and convolved with a gaussian hemodynamic response function at a peak time of $6.5 \mathrm{~s}$. Time series were analysed by applying a general linear model approach [49] using Matlab Version 7 software (MathWorks Inc.). The resulting $\beta$ estimates per condition and subject served as parameter set for subsequent testing. According to our hypothesis of an increasing $\mathrm{O}_{2} \mathrm{Hb}$ concentration towards early and late extinction trials for CS+ compared to CS-, we determined differential $\beta$ values for CS+ and CS- each and contrasted these differences by using paired t tests: $\left[\left(\mathrm{CS}_{+_{\text {late }}}-\mathrm{CS}+_{\text {early }}\right)-\left(\mathrm{CS}_{-{ }_{-} \text {ate }}-\mathrm{CS}_{-}{ }_{\text {early }}\right)\right]$. Correction for multiple comparisons across probe set channels were performed by using a cluster permutation approach. Specifically, we compared the cluster size of significantly active channels (at $\mathrm{p}<0.1$ for each channel) to the distribution of cluster sizes expected under the null hypothesis (adapted for the 2-d fNIRS case from Wager et al. [50]). To obtain the null distribution, we performed 10,000 permutation tests across all channels given a single channel $\mathrm{p}$ value $<0.1$. Activation was thus considered significant if the probability of obtaining this cluster size under the null hypothesis was $\mathrm{p}<0.05$. According to a probabilistic map (http:// www.jichi.ac.jp/brainlab/virtual_regE.html\#AnatomLabel) we provide MNI coordinates ( $\mathrm{x}, \mathrm{y}, \mathrm{z}$ ) of significant fNIRS channels to allow for integration of our results across imaging methods.

SCR and fNIRS data were tested for significant correlations using Pearson's correlation coefficient and one-tailed tests due to our expectations of negative correlations between SCR and $\mathrm{O}_{2} \mathrm{Hb}$ within fNIRS channels or even channel clusters during the extinction phases. For exploratory purposes, we examined the influence of expectancy and SCR as well as fNIRS data to consider the influence of prediction error (two-tailed tests). 


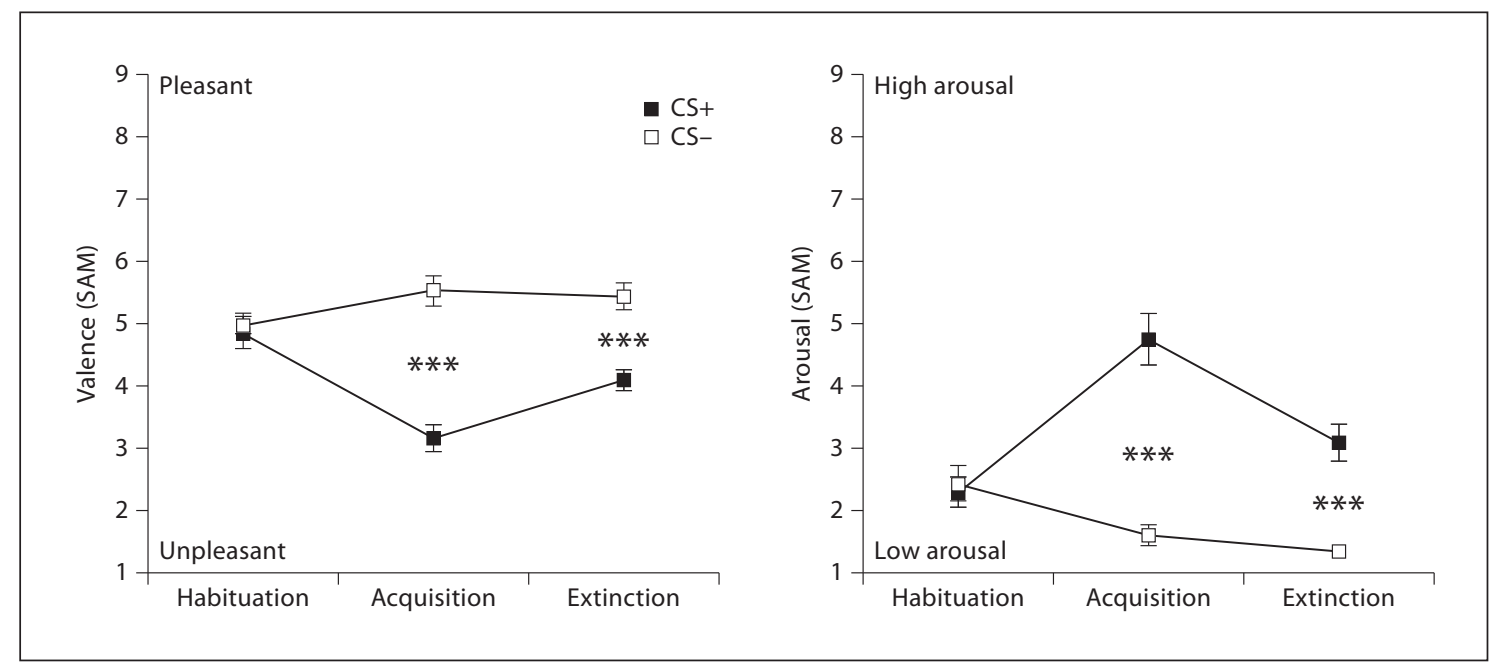

Fig. 2. Valence and arousal ratings. Assessment by using the Self-Assessment Manikin (SAM [42]). Significantly different values for CS+ and CS- per experimental phase (mean + SEM) are depicted as *** to indicate a significance level of $\mathrm{p}<0.001$.

\section{Results}

As expected, subjects rated the UCS as quite unpleasant (mean 7.8, SD 1.3, range 4-10) indicating that the scream of $95 \mathrm{~dB}$ was aversive enough to induce fear conditioning. All 34 participants reported awareness of the CS-UCS contingency at the end of the acquisition phase and displayed a significant linear decrement of the UCS expectancy ratings during the extinction phase (linear trend test: $F(1,33)=24.53, \mathrm{p}<0.001)$ from $78 \%$ after the first third, over $62 \%$ after the second third to $52 \%$ at the end of the experiment. Concerning the valence and arousal ratings, the repeated-measures ANOVA yielded main effects for stimulus (valence: $F(1,33)=29.37$, $\mathrm{p}<$ 0.001; arousal: $F(1,33)=43.25, \mathrm{p}<0.001)$ and phase (valence: $F(1.5,52.7)=15.33, \mathrm{p}<0.001$; arousal: $F(1.5$, $50.8)=13.98, \mathrm{p}<0.001)$ as well as significant stimulus $\times$ phase interactions (valence: $F(1.3,42.3)=23.49, \mathrm{p}<0.001$; arousal: $F(1.3,41.4)=34.02, \mathrm{p}<0.001)$. As expected, post hoc $t$ tests revealed that CS+ and CS- were equally evaluated after the habituation phase, both as neutral and sparsely arousing, but were differentially rated during conditioning. Herein, CS+ ratings were significantly lower in valence $\left(t_{33}=6.51, \mathrm{p}<0.001\right)$ and higher in arousal than for CS $-\left(t_{33}=7.52, \mathrm{p}<0.001\right)$. Comparing acquisition and extinction phase, the decrement for CS+ arousal and the increase for CS+ valence became significant (arousal: $t_{33}=7.02, \mathrm{p}<0.001$; valence: $t_{33}=6.5, \mathrm{p}<0.001$ ) although the differential ratings persisted during extinc- tion learning (valence: $t_{33}=5.55, \mathrm{p}<0.001$; arousal: $t_{33}=$ 6.13, p < 0.001; fig. 2).

For SCR analyses we had to exclude 2 female subjects who did not display any fluctuations in their responses to either CS or UCS across the whole experiment. Analyses of the remaining sample of 32 subjects revealed similar results as for subjective ratings indicating a successful conditioning during acquisition and additionally suggesting extinction learning within the last experimental phases (fig. 3). The $2 \times 4$ repeated-measures ANOVA showed significant main effects for stimulus $(F(1,31)=$ $15.82, \mathrm{p}<0.001)$ as well as phase $(F(3,93)=6.74, \mathrm{p}<0.001)$ and again a significant interaction $(F(3,93)=4.51, \mathrm{p}=$ $0.005)$. Post hoc $t$ tests demonstrated significantly higher SCR amplitudes to CS+ than CS- during acquisition $\left(t_{31}=4.87, \mathrm{p}<0.001\right)$, which significantly diminished during the extinction phases (paired t test for SCR to CS+ during acquisition compared to early extinction: $t_{31}=$ 4.15, $\mathrm{p}<0.001$, and late extinction: $t_{31}=3.77, \mathrm{p}<0.001$ ). Differences between CS+ and CS- remained significant during the time course of extinction (early extinction: $t_{31}=1.93, \mathrm{p}=0.032$; late extinction: $t_{31}=2.5, \mathrm{p}=0.009$ ). However, SCR amplitudes during the extinction phase returned to habituation level (early extinction: $t_{31}=0.55$, $\mathrm{p}=0.293$; late extinction: $t_{31}=0.69, \mathrm{p}=0.248$ ) and further did not change significantly through early and late extinction trials $\left(t_{31}=0.33, \mathrm{p}=0.746\right)$.

For the fNIRS data we did not have to exclude channels from analyses due to little motion artefacts in the 


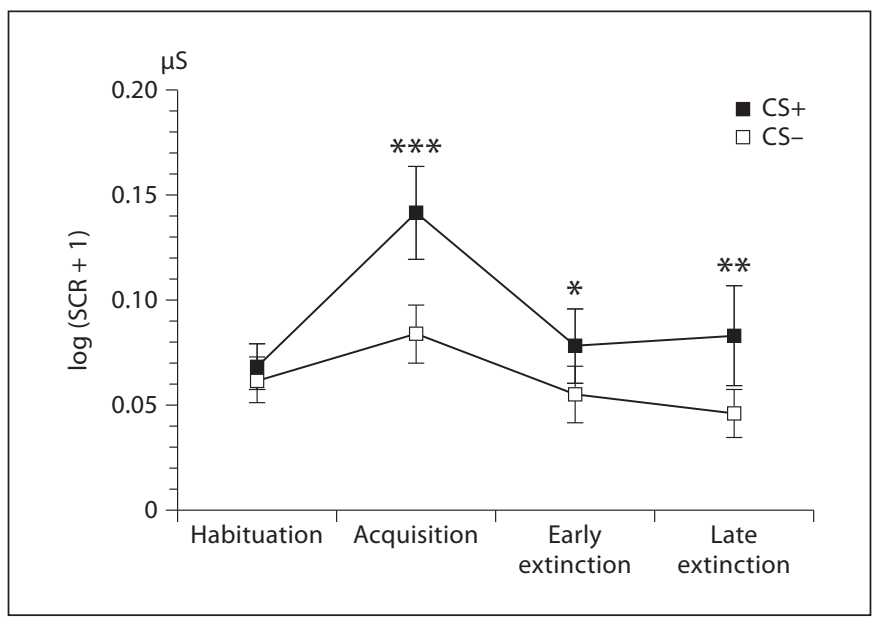

Fig. 3. ANOVA results for SCR to CS+ and CS-. As expected, SCR to $\mathrm{CS}+$ trials increased during conditioning and decreased during extinction indicating successful conditioning and extinction. Responses to the CS-remained unchanged through the experiment. ${ }^{*} \mathrm{p}<0.05,{ }^{* *} \mathrm{p}<0.01,{ }^{* * *} \mathrm{p}<0.001$.

frontal region as expected. We found four significant probe set channels for which our analysed $t$-contrast $\left[\left(\mathrm{CS}+_{\text {late }}-\mathrm{CS}+_{\text {early }}\right)-\left(\mathrm{CS}-_{\text {late }}-\mathrm{CS}_{-}\right.\right.$early $\left.\left._{\mathrm{y}}\right)\right]$ revealed significant results, that is the difference between early and late trials was larger for CS+ than CS- (channel 35: $t_{33}=$ $2.26, \mathrm{p}=0.016[\mathrm{x}=27, \mathrm{y}=68, \mathrm{z}=9]$; channel $27: t_{33}=2.22$, $\mathrm{p}=0.017[\mathrm{x}=-13, \mathrm{y}=68, \mathrm{z}=20]$; channel 26: $t_{33}=1.92$, $\mathrm{p}=0.032[\mathrm{x}=15, \mathrm{y}=68, \mathrm{z}=21] ;$ channel $47: t_{33}=1.79$, $\mathrm{p}=0.042[\mathrm{x}=15, \mathrm{y}=71, \mathrm{z}=-3])$. Eleven other channels revealed significant results by trend and are mentioned for completeness (channel 20: $t_{33}=1.64, \mathrm{p}=0.055$; channel 16: $t_{33}=1.62, \mathrm{p}=0.057$; channel $24: t_{33}=1.62, \mathrm{p}=$ 0.058; channel 36: $t_{33}=1.61, \mathrm{p}=0.059$; channel 19: $t_{33}=$ $1.58, \mathrm{p}=0.062$; channel $21: t_{33}=1.57, \mathrm{p}=0.066$; channel 5: $t_{33}=1.57, \mathrm{p}=0.063$; channel $37: t_{33}=1.53, \mathrm{p}=0.068$; channel 29: $t_{33}=1.5, \mathrm{p}=0.072$; channel $45: t_{33}=1.42, \mathrm{p}=$ 0.083; channel 51: $t_{33}=1.4, \mathrm{p}=0.086$ ). Ten of the aforementioned probe set channels resulted in one single significant cluster ( $\mathrm{p}<0.03$; channels $35,27,26,47,16,24$, $36,5,37,45)$; no other cluster reached the significance threshold. Figure 4 pictures mean $\beta$ values for this cluster by separating into CS+ and CS- as well as early and late trials according to our analysed $t$-contrast (see above). The cluster is being composed of a significant increase of $\mathrm{CS}+$ trials during the early and late extinction phase $\left(t_{33}=1.89, \mathrm{p}=0.034\right)$ and significant exceeding $\mathrm{O}_{2} \mathrm{Hb}$ values between CS+ and CS- trials during the late extinction phase $\left(t_{33}=2.2, \mathrm{p}=0.018\right)$. We neither found differences between CS - trials across the two phases $\left(t_{33}=0.9\right.$,

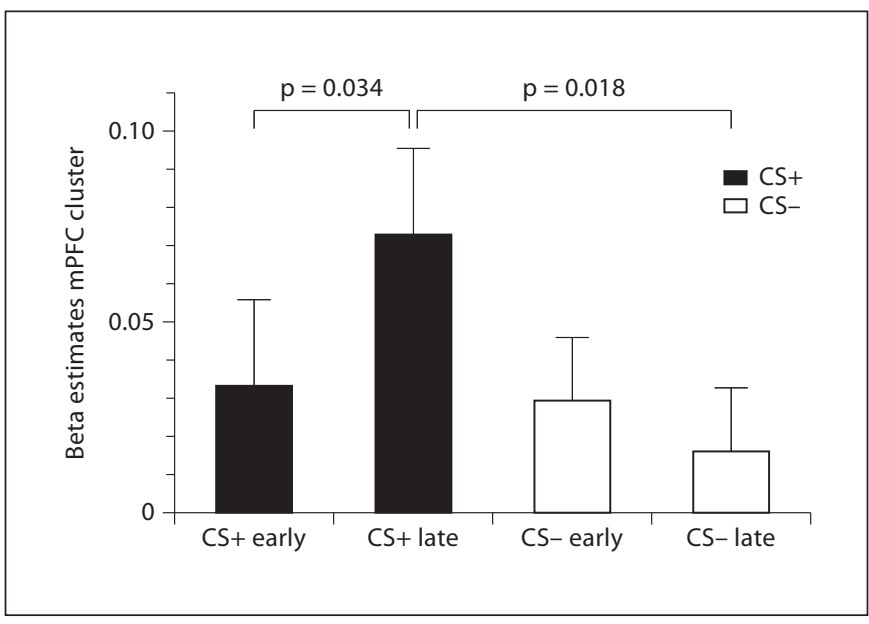

Fig. 4. Beta estimates of the regressors CS+ early, CS+ late, CSearly and CS- late. Depicted are cluster means and standard errors of the cluster means.

$\mathrm{p}=0.187)$ nor between CS + and CS - early trials $\left(t_{33}=\right.$ $0.23, \mathrm{p}=0.41$; fig. 4,5 ).

As depicted in figure $5 b$, the hemodynamic responses approximately started according to the expected UCS onset (around 5,000 ms) and revealed no differences during CS presentation. Hemodynamic responses starting at the expected UCS onset during extinction learning seem to reflect a prediction error, i.e. the expected UCS did not occur.

\section{Correlations}

Correlation analyses concerning SCR and $\mathrm{O}_{2} \mathrm{Hb}$ in the sample of 32 subjects revealed a negative correlation between mean cluster $\mathrm{O}_{2} \mathrm{Hb}$ values for late CS+ trials and the difference score between early and late SCR to CS+ $(r=-0.327 ; \mathrm{p}=0.034)$. Subjects who displayed decreasing SCR to CS+ from early to late trials as it is expected during successful extinction, showed higher $\beta$ values within late extinction phase indicating higher activity within the mPFC. In order to adequately compare SCR and fNIRS data during the same time interval, we additionally performed SCR peak detection during the extinction phase in a later interval between 5.5 and $8.5 \mathrm{~s}$ after CS presentation. This segment corresponded to the analysed fNIRS segment, which was assumed to mark the hemodynamic response function onset predicting the UCS. Even if we changed the analysed SCR segment in this way, the negative correlation between the difference score of late and early SCR responses and the cluster activity during the 


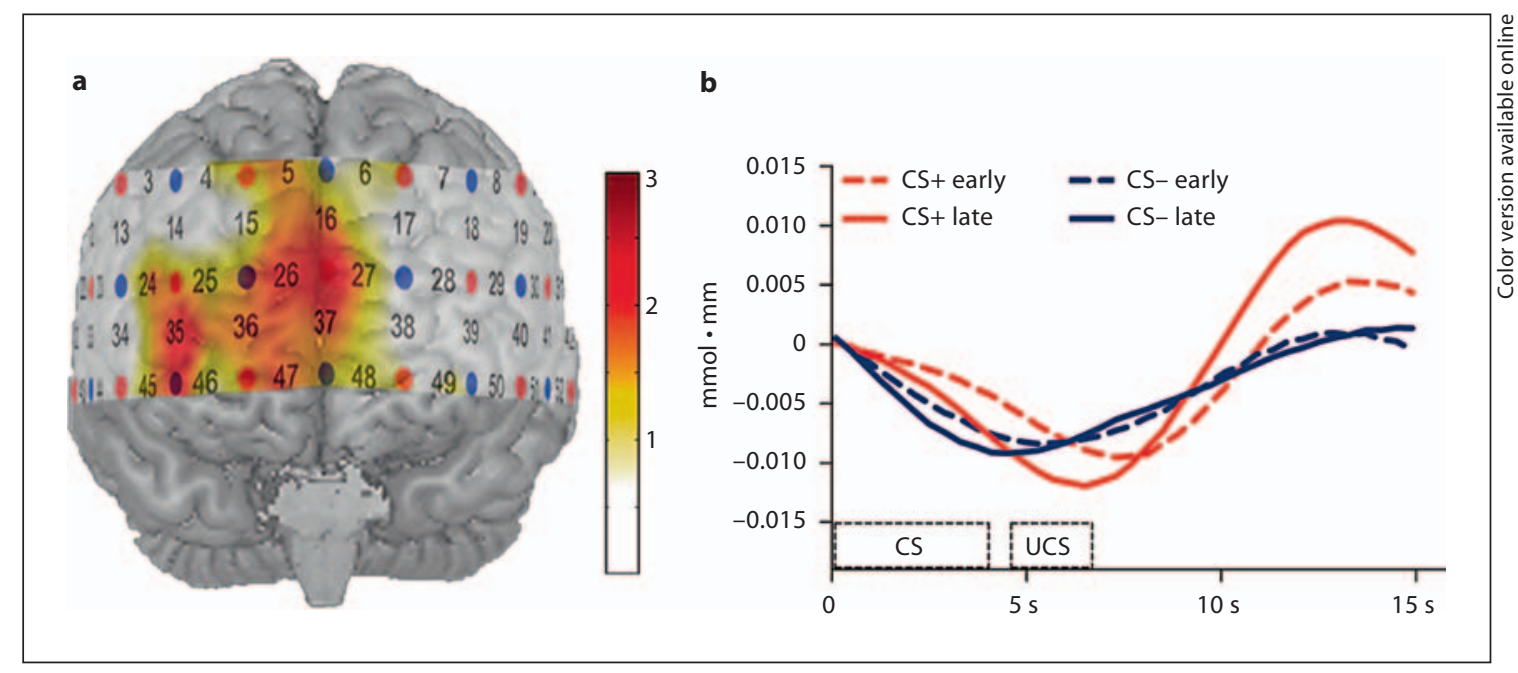

Fig. 5. a T-map superimposed on a standard brain. The comparison 'CS+ late vs. CS+ early' revealed significant $\mathrm{O}_{2} \mathrm{Hb}$ concentration changes in the mPFC cluster at a peak time of $6.5 \mathrm{~s}$ after CS+ offset. $\mathbf{b}$ Time series of all regressors.

late extinction phase remained significant $(r=-0.324$; $\mathrm{p}=0.035)$. The exploratory analyses concerning the recurrence of the UCS revealed that only the second out of three expectancy ratings correlated significantly with SCR to CS+ trials within the early $(r=0.37, \mathrm{p}=0.037)$ and late extinction phase $(r=0.4, \mathrm{p}=0.022)$, suggesting that subjects who subjectively tend to resist to extinction learning show appropriately higher SCR values during the extinction phases and herewith demonstrate less well extinction learning than participants who did report more certainty towards the disappearance of the UCS.

\section{Discussion}

In the present study, 34 healthy subjects underwent a fear conditioning paradigm with two neutral faces as CS and a loud, aversive scream as UCS to examine the time course of extinction learning by analysing concentration changes in $\mathrm{O}_{2} \mathrm{Hb}$ across early and late extinction trials. SCR and valence as well as arousal ratings were assessed to ensure successful conditioning.

We found significantly different valence and arousal ratings for CS+ and CS- trials as well as SCR data after the habituation phase. CS+ presentations evoked lower valence ratings and appropriately higher arousal ratings for CS+ than CS- as well as higher SCR amplitudes. During the extinction phase, fNIRS data displayed a significant increase in response to CS+ trials from early to late extinction within one cluster of 10 probe set channels covering the mPFC. The cluster activity elicited by CS+ trials further exceeded CS- trials during late extinction while $\beta$ values for CS- showed no significant difference across both extinction phases. To our knowledge, this is the first study investigating fear extinction by using fNIRS. This optical imaging method is restricted to the cortical surface and therefore cannot directly be compared to methods with higher spatial resolution, e.g. fMRI. Nonetheless, our findings are in accordance with previous imaging results confirming an $\mathrm{mPFC}$ contribution during within-session extinction [e.g. 25, 26, 36].

In order to critically review our results, we have to mention some inconsistencies of the data and will follow to discuss these aspects in light of the current literature. First one might argue that valence and arousal ratings as well as SCR data did not reflect successful extinction learning. We did find strong conditioning effects for all variables, but irrespective of the UCS absence during the extinction phase, participants continued to rate the former CS+ as significantly more unpleasant and more arousing than the CS-. However, there are other studies that found this kind of resistance to extinction in verbal reports [e.g. 25-28], and besides, UCS expectancy ratings showed a constantly decreasing expectancy across the extinction phase, reflecting that our participants did unlearn the CS-UCS association. Differential verbal ratings for CS+ and CS- might reflect lasting aversiveness of the UCS that prolonged throughout extinction trials, but on 
the physiological level we do see an altered fear processing. SCR levels during early and late extinction decreased significantly from acquisition and further reached the habituation level as it is defined for successful extinction learning. Above all, at the rate of extinction and acquisition the number of trials is comparable to former studies $[23,27]$ or even contains a higher number of extinction trials [25-28].

Another constraint of our study is related to the mPFC activity we associated with extinction learning. A recently published review by Etkin et al. [51] argued that $\mathrm{mPFC}$ activity during extinction learning might reflect remnants of fear conditioning because studies on fear appraisal and sympathetic arousal also found $\mathrm{mPFC}$ engagement while generating fear responses. If $\mathrm{mPFC}$ activity would indeed reflect an explicit threat evaluation, one would have expected a decrease in activity from early to late trials contrarily to the increasing $\mathrm{mPFC}$ activity we found in the present study. Beta values for CS+ and CS-also started on an equal level during early extinction, we thus argue that mPFC activity in our study reflects extinction learning rather than a fear response. This is in line with the already explained successful induction of extinction, immanent in our UCS expectancy ratings and SCR data. Moreover, correlations between $\mathrm{SCR}$ and $\mathrm{O}_{2} \mathrm{Hb}$ values emphasise the expected top-down control executed by the $\mathrm{MPFC}$ as subjects who exhibited a greater SCR decrement from early to late extinction phase also revealed higher $\beta$ values and thereby more activity in the $\mathrm{mPFC}$ during the late $\mathrm{CS}+$ condition. A study investigating fear conditioning as a form of prediction error learning does also confirm our assumption. Spoormaker et al. [52] examined CS+ trials in which no UCS was administered and found increased activity in ventromedial, dorsolateral and orbitofrontal regions as neuronal correlates of this so-called negative prediction error. The absence of negative consequences therefore seems to be associated with prefrontal engagement that would also fit explanations of fear extinction [52]. This might also explain the timing of our hemodynamic response function. The temporal gap between CS+ and UCS presentations enabled us to examine the onset of the expected neuronal response towards the anticipated UCS during the extinction phase rather than the CS onset. The mentioned negative prediction error is existent if the UCS did not occur against one's expectation. This mPFC-coupled learning process could only start in the absence of the UCS and not in the beginning of the CS presentation. Linnman et al. [53] investigated neuronal responses on shock delivery in a fear conditioning paradigm and found increased engagement of the dorsal anterior cingulate cor- tex, a region corresponding to the $\mathrm{mPFC}$, during the nondelivery of an expected UCS. This finding fits our results as well, although it restricts comparability to available studies on fear extinction that did not provide such temporal information about CS and UCS. Future studies taking these differences into account would certainly contribute to a better understanding of temporal interactions such as the functional mPFC-amygdala coupling.

One major limitation of our study is the restricted application of fNIRS during the extinction phase. We discussed our result of significant $\mathrm{mPFC}$ activity as successful extinction learning, but in fact we cannot strictly obviate $\mathrm{mPFC}$ contribution during the acquisition phase. Numerous animal studies highlighted prefrontal contribution during extinction learning, i.e. when the CR is already acquired. Moreover, a systematic review about neuroimaging literature on human fear conditioning by Sehlmeyer et al. [12] did not find support for mPFC involvement during fear conditioning. Thus, it appears reasonable to restrict our fNIRS measurement exclusively to the extinction phase.

Secondly, we have to admit that we did not investigate $\mathrm{mPFC}$ activity during extinction retention, i.e. $24 \mathrm{~h}$ after the initial fear conditioning. The present study was not intended to compare mPFC engagement on the acquisition and recall of extinction. We well know that the ventromedial prefrontal cortex frequently found in animal studies is involved in recall of extinction rather than the initial acquisition [16], on the other hand there are the aforementioned studies in humans that found MPFC activity already during within-session extinction. It might be possible that long-term storage of extinction memory is supported in other for example more dorsal situated brain regions as Gottfried and Dolan [24] already speculated. Future studies therefore have to consider the consolidation of extinction memory by implementing a second extinction training after a delay period.

A third limitation relates to context changes which might have occurred through attaching the fNIRS probe set. In this connection we have to consider context dependency of extinction learning suggesting a return of fear by presenting the formerly CS+ again in the initial context, i.e. without the fNIRS probe set. We would again like to stress the fact that we kept all other parameters constant during the short break for attaching the probe set to minimize context effects. However, future studies using fNIRS for investigations of prefrontal activation during extinction learning could overcome this limitation by implementing shorter fear conditioning paradigms to assess all experimental phases. 


\section{Conclusions}

The present study revealed increasing MPFC activation to CS+ trials during extinction that was different from that for a CS- which displayed no change across early and late extinction learning. Based on these findings, we propose $\mathrm{mPFC}$ activity during extinction learning to reflect better regulation of CR expression. The increase of prefrontal contribution from early to late extinction trials seems to be associated with changes in the associative significance of CS+ and UCS. Increasing associative strength might thereby rely on amygdala activity, decreasing associative strength appears to be inversely correlated with mPFC activity. Patients suffering from anxiety disorders or even high trait-anxious subjects have been characterized by increased CR to threat cues and reduced extinction [54]. Thus, they show deficient associative learning and accordingly deficient recruitment of amygdala and $\mathrm{mPFC}[26,28]$.

Future studies have to examine cortical-subcortical interactions in more detail to ascertain strategies to affect mPFC activity in the treatment of anxiety disorders. Combined methods such as fNIRS and fMRI would fur- ther provide complementary results [44] by offering both high temporal as well as high spatial resolution. Here, a more precise definition of mPFC subregions involved in extinction learning might open up prospects to strengthen prefrontal areas, and transcranial magnetic stimulation could for instance be such a tool [55]. Transcranial magnetic stimulation is as restricted to the cortical surface as fNIRS. The use of fNIRS for mapping the prefrontal cortex is therefore not contradictory by searching innovative treatment options for facilitating extinction learning or even exposure therapy.

\section{Acknowledgments}

The authors would like to thank Juliana Rost for her support in participant recruiting and data acquisition. We also thank Michael M. Plichta for his ideas in data analyses and modelling, Evelyn Glotzbach who helped us with analysing the SCR data as well as Wilma Harnisch for calibrating our sound system.

This publication was funded by the German Research Foundation (DFG, SFB TRR 58, C04 project). The funding source had no role in study design, data collection and analyses, decision to publish, or preparation of the manuscript.

\section{References}

1 Hamm AO, Weike AI: The neuropsychology of fear learning and fear regulation. Int J Psychophysiol 2005;57:5-14.

2 Myers KM, Davis M: Behavioral and neural analysis of extinction. Neuron 2002;36:567584.

- 3 Maren S, Quirk GJ: Neuronal signalling of fear memory. Nat Rev Neurosci 2004;5:844852.

4 Bouton ME: Context, ambiguity, and unlearning: sources of relapse after behavioral extinction. Biol Psychiatry 2002;52:976-986.

5 Garcia R, Vouimba RM, Baudry M, Thompson RF: The amygdala modulates prefrontal cortex activity relative to conditioned fear. Nature 1999;402:294-296.

-6 Morgan MA, Romanski LM, LeDoux JE: Extinction of emotional learning: Contribution of medial prefrontal cortex. Neurosci Let 1993;163:109-113.

7 Rosenkranz JA, Moore H, Grace AA: The prefrontal cortex regulates lateral amygdala neuronal plasticity and responses to previously conditioned stimuli. J Neurosci 2003; 23:11054-11064.

$>8$ Herry C, Ciocchi S, Senn V, Demmou L, Muller C, Luthi A: Switching on and off fear by distinct neuronal circuits. Nature 2008; 454:600-606
\$9 Quirk GJ, Likhtik E, Pelletier JG, Pare D: Stimulation of medial prefrontal cortex decreases the responsiveness of central amygdala output neurons. J Neurosci 2003;23: 8800-8807.

10 Milad MR, Vidal-Gonzalez I, Quirk GJ: Electrical stimulation of medial prefrontal cortex reduces conditioned fear in a temporally specific manner. Behav Neurosci 2004; 118:389-394.

11 Pape HC, Pare D: Plastic synaptic networks of the amygdala for the acquisition, expression, and extinction of conditioned fear. Physiol Rev 2010;90:419-463.

12 Sehlmeyer C, Schoning S, Zwitserlood P, Pfleiderer B, Kircher T, Arolt V, Konrad C: Human fear conditioning and extinction in neuroimaging: a systematic review. PLoS One 2009;4:e5865.

13 Phillips RG, LeDoux JE: Differential contribution of amygdala and hippocampus to cued and contextual fear conditioning. Behav Neurosci 1992;106:274-285.

14 Bechara A, Tranel D, Damasio H, Adolphs R, Rockland C, Damasio AR: Double dissociation of conditioning and declarative knowledge relative to the amygdala and hippocampus in humans. Science 1995;269:1115-1118.
15 Weible AP, McEchron MD, Disterhoft JF: Cortical involvement in acquisition and extinction of trace eyeblink conditioning. Behav Neurosci 2000;114:1058-1067.

16 Quirk GJ, Russo GK, Barron JL, Lebron K: The role of ventromedial prefrontal cortex in the recovery of extinguished fear. J Neurosci 2000;20:6225-6231.

17 Barrett D, Shumake J, Jones D, Gonzalez-Lima F: Metabolic mapping of mouse brain activity after extinction of a conditioned emotional response. J Neurosci 2003;23:5740-5749.

18 Buchel C, Dolan RJ: Classical fear conditioning in functional neuroimaging. Curr Opin Neurobiol 2000;10:219-223.

19 Buchel C, Morris J, Dolan RJ, Friston KJ: Brain systems mediating aversive conditioning: AN event-related fMRI study. Neuron 1998;20:947-957.

-20 LaBar KS, Gatenby JC, Gore JC, LeDoux JE, Phelps EA: Human amygdala activation during conditioned fear acquisition and extinction: a mixed-trial fMRI study. Neuron 1998; 20:937-945.

21 Buchel C, Dolan RJ, Armony JL, Friston KJ Amygdala-hippocampal involvement in human aversive trace conditioning revealed through event-related functional magnetic resonance imaging. J Neurosci 1999;19: 10869-10876. 
-22 Knight DC, Smith CN, Cheng DT, Stein EA, Helmstetter FJ: Amygdala and hippocampal activity during acquisition and extinction of human fear conditioning. Cogn Affect Behav Neurosci 2004;4:317-325.

-23 Phelps EA, Delgado MR, Nearing KI, LeDoux JE: Extinction learning in humans: role of the amygdala and vmPFC. Neuron 2004;43:897-905.

24 Gottfried JA, Dolan RJ: Human orbitofrontal cortex mediates extinction learning while accessing conditioned representations of value. Nat Neurosci 2004;7:1144-1152.

-25 Lang S, Kroll A, Lipinski SJ, Wessa M, Ridder S, Christmann C, Schad LR, Flor H: Context conditioning and extinction in humans: differential contribution of the hippocampus, amygdala and prefrontal cortex. Eur J Neurosci 2009;29:823-832.

26 Sehlmeyer C, Dannlowski U, Schoning S, Kugel H, Pyka M, Pfleiderer B, Zwitserlood P, Schiffbauer H, Heindel W, Arolt V, Konrad C: Neural correlates of trait anxiety in fear extinction. Psychol Med 2010:1-10.

-27 Reinhardt I, Jansen A, Kellermann T, Schuppen A, Kohn N, Gerlach AL, Kircher T: Neural correlates of aversive conditioning: development of a functional imaging paradigm for the investigation of anxiety disorders. Eur Arch Psychiatry Clin Neurosci 2010;260: 443-453.

28 Barrett J, Armony JL: Influence of trait anxiety on brain activity during the acquisition and extinction of aversive conditioning. Psychol Med 2009;39:255-265.

29 Hugdahl K, Berardi A, Thompson WL, Kosslyn SM, Macy R, Baker DP, Alpert NM, LeDoux JE: Brain mechanisms in human classical conditioning: a pet blood flow study. Neuroreport 1995;6:1723-1728.

-30 Milad MR, Wright CI, Orr SP, Pitman RK, Quirk GJ, Rauch SL: Recall of fear extinction in humans activates the ventromedial prefrontal cortex and hippocampus in concert. Biol Psychiatry 2007;62:446-454.

- 31 Kalisch R, Korenfeld E, Stephan KE, Weiskopf N, Seymour B, Dolan RJ: Context-dependent human extinction memory is mediated by a ventromedial prefrontal and hippocampal network. J Neurosci 2006;26: 9503-9511.

- 32 Indovina I, Robbins TW, Núñez-Elizalde AO, Dunn BD, Bishop SJ: Fear-conditioning mechanisms associated with trait vulnerability to anxiety in humans. Neuron $2011 ; 69$ : 563-571.
3 Bishop S, Duncan J, Brett M, Lawrence AD: Prefrontal cortical function and anxiety: Controlling attention to threat-related stimuli. Nat Neurosci 2004;7:184-188.

-34 Hoshi Y, Kobayashi N, Tamura M: Interpretation of near-infrared spectroscopy signals: a study with a newly developed perfused rat brain model. J Appl Physiol 2001;90:16571662 .

35 Herrmann MJ, Ehlis AC, Fallgatter AJ: Prefrontal activation through task requirements of emotional induction measured with NIRS. Biol Psychol 2003;64:255-263.

36 Molchan SE, Sunderland T, McIntosh AR, Herscovitch P, Schreurs BG: A functional anatomical study of associative learning in humans. Proc Natl Acad Sci USA 1994;91: 8122-8126.

37 Sheehan DV, Lecrubier Y, Sheehan KH, Amorim P, Janavs J, Weiller E, Hergueta T, Baker R, Dunbar GC: The Mini-International Neuropsychiatric Interview (MINI): the development and validation of a structured diagnostic psychiatric interview for DSM-IV AND ICD-10. J Clin Psychiatry 1998;59 (suppl 20):22-33, quiz 34-57.

38 Oldfield RC: The assessment and analysis of handedness: The Edinburgh Inventory. Neuropsychologia 1971;9:97-113.

39 Milad MR, Goldstein JM, Orr SP, Wedig MM, Klibanski A, Pitman RK, Rauch SL: Fear conditioning and extinction: influence of sex and menstrual cycle in healthy humans. Behav Neurosci 2006;120:1196-1203.

-40 Tottenham N, Tanaka JW, Leon AC, McCarry T, Nurse M, Hare TA, Marcus DJ, Westerlund A, Casey BJ, Nelson C: The NimStim set of facial expressions: judgments from untrained research participants. Psychiatry Res 2009; 168:242-249.

41 Bradley MM, Lang PJ: International Affective Digitized Sounds (IADS): Stimuli, Instruction Manual and Affective Ratings (Tech. Rep. No. B-2). Gainesville, The Center for Research in Psychophysiology, University of Florida, 1999.

42 Bradley MM, Lang PJ: Measuring emotion the Self-Assessment Manikin and the semantic differential. J Behav Ther Exp Psychiatry 1994;25:49-59.

43 Critchley HD: Electrodermal responses: what happens in the brain. Neuroscientist 2002;8:132-142.
44 Hoshi Y: Towards the next generation of near-infrared spectroscopy. Philos Transact A Math Phys Eng Sci 2011;369:4425-4439.

45 Obrig H, Villringer A: Beyond the visible imaging the human brain with light. J Cereb Blood Flow Metab 2003;23:1-18.

46 Hoshi Y: Functional near-infrared spectroscopy: current status and future prospects. J Biomed Opt 2007;12:062106.

-47 Okamoto M, Dan H, Sakamoto K, Takeo K, Shimizu K, Kohno S, Oda I, Isobe S, Suzuki T, Kohyama K, Dan I: Three-dimensional probabilistic anatomical cranio-cerebral correlation via the International $10-20$ system oriented for transcranial functional brain mapping. Neuroimage 2004;21:99111.

48 Hoshi Y, Shimada M, Sato C, Iguchi Y: Reevaluation of near-infrared light propagation in the adult human head: implications for functional near-infrared spectroscopy. J Biomed Opt 2005; 10:064032.

49 Plichta MM, Heinzel S, Ehlis AC, Pauli P, Fallgatter AJ: Model-based analysis of rapid event-related functional near-infrared spectroscopy (NIRS) data: a parametric validation study. Neuroimage 2007;35:625-634.

50 Wager TD, Scott DJ, Zubieta J-K: Placebo effects on human $\mu$-opioid activity during pain. Proc Natl Acad Sci USA 2007;104: 11056-11061.

-51 Etkin A, Egner T, Kalisch R: Emotional processing in anterior cingulate and medial prefrontal cortex. Trends Cogn Sci 2011;15:8593.

-52 Spoormaker VI, Andrade KC, Schröter MS Sturm A, Goya-Maldonado R, Sämann PG, Czisch M: The neural correlates of negative prediction error signaling in human fear conditioning. Neuroimage 2011;54:22502256.

53 Linnman C, Rougemont-Bücking A, Beucke JC, Zeffiro TA, Milad MR: Unconditioned responses and functional fear networks in human classical conditioning. Behav Brain Res 2011:221:237-245.

-54 Lissek S, Powers AS, McClure EB, Phelps EA Woldehawariat G, Grillon C, Pine DS: Classical fear conditioning in the anxiety disorders: a meta-analysis. Behav Res Ther 2005; 43:1391-1424.

55 Milad MR, Quirk GJ: Neurons in medial prefrontal cortex signal memory for fear extinction. Nature 2002;420:70-74. 